\title{
Design and Optimization of Elliptical Cavity Tube Receivers in the Parabolic Trough Solar Collector
}

\author{
Fei Cao, ${ }^{1,2}$ Lei Wang, and Tianyu $\mathrm{Zhu}^{1}$ \\ ${ }^{1}$ College of Mechanical and Electrical Engineering, Hohai University, Changzhou 213022, China \\ ${ }^{2}$ Sunshore Solar Energy Company Limited, Nantong 226300, China \\ Correspondence should be addressed to Fei Cao; yq.cao@hotmail.com
}

Received 8 January 2017; Accepted 28 February 2017; Published 30 March 2017

Academic Editor: Stoian Petrescu

Copyright (C) 2017 Fei Cao et al. This is an open access article distributed under the Creative Commons Attribution License, which permits unrestricted use, distribution, and reproduction in any medium, provided the original work is properly cited.

\begin{abstract}
The nonfragile cavity receiver is of high significance to the solar parabolic trough collector (PTC). In the present study, light distributions in the cavity under different tracking error angles and PTC configurations are analyzed. A new elliptical cavity geometry is proposed and analyzed. It is obtained from this study that light distribution on the tube receiver is asymmetrical when tracking error occurs. On increasing the tracking error angle, more lights are sheltered by the cavity outer surface. The PTC focal distance has negative correlation with the cavity open length, whereas the PTC concentration ratio has positive correlation with the cavity open length. Increasing the tracking error angle and increasing the PTC focal distance would both decrease the cavity blackness. Introducing a flat plate reflector at the elliptical cavity open inlet can largely increase the cavity darkness.
\end{abstract}

\section{Introduction}

Solar energy has been recognized as one of the most important energy sources at present and in the further energy structure. Due to the discontinuous, low energy flux, periodicity, and unsteady characteristics of solar energy, solar concentration is commonly utilized in solar engineering fields, among which the solar parabolic trough collectors (PTC) are the most widely accepted solar concentration style [1]. The light ray tracing [2-4] and structural analysis [5-7] for the PTC have been widely discussed in the literature.

Serrano-Aguilera et al. studied the continuous linear reflectors for flat plate receivers with Inverse Monte Carlo ray-tracing method, where a quasi-planar-concentrated flux distribution is required [2]. Cheng et al. made a 3D numerical study of heat transfer characteristics in the receiver tube of PTC [3], and they then carried out a comparative analysis for PTC with a detailed Monte Carlo ray-tracing optical model [4]. They concluded that the ideal characteristics and optical performance of the PTCs were very different from some critical points determined by the divergence phenomenon of the nonparallel solar beam [4]. Giannuzzi et al. defined a guideline for steel structures' design and assessment of the components of PTC. Their codes were developed for practical usage and were evaluated under some specific conditions [5]. Liang et al. compared three optical models for the PTC and optimized the geometric parameters according to their models [6]. Cheng et al. optimized the geometrical structure of the PTC based on the particle swarm optimization algorithm and the Monte Carlo ray-tracing method, which found a balance between the calculating speed and result accuracy [7].

With respect to the heat receiver of the PTC, some structures are designed for the PV or PV/T in PTCs [3]. However, tube receivers are more commonly used in PTC combined with thermodynamic cycles [8-10]. In general, two kinds of tubes are commonly utilized in the PTC as the receiver, namely, the vacuum tube $[2,4-10]$ and the cavity tube [11-13]. The main advantages of the vacuum tube are the high thermal maintenances and low module cost, whereas its structure is frangible. Though a kind of "metal inner and vacuum glass outer" tube is proposed, the connection area of metal and glass is also frangible due to 


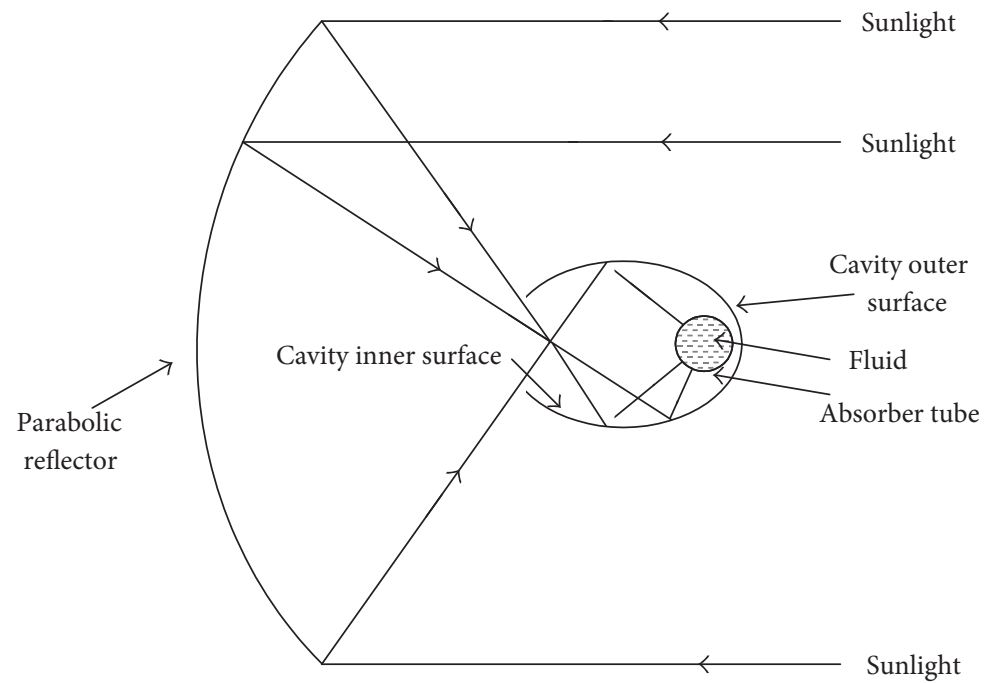

FIgURE 1: Schematic of a cavity tube receiver in the PTC.

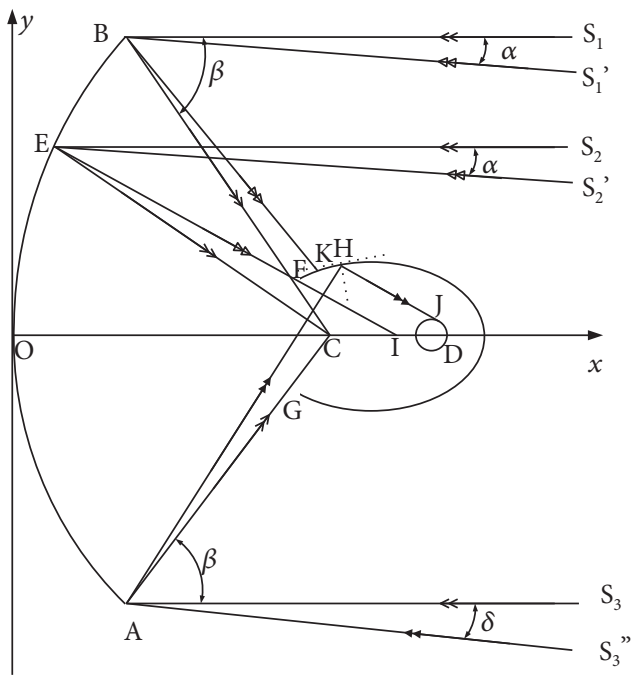

FIgure 2: Sunlight trace in a PTC with an elliptical cavity tube receiver by considering the tracking error angle $\alpha$ under the rectangular coordinate system.

uninterrupted and periodical thermal stress from the inner and outer sides. The other kind of solar receiver is the cavity tube, whose schematic is shown in Figure 1. The outer cover of the receiver is the cavity, with an open inlet towards the parabolic trough reflector. As there is no connection area between the cavity outer cover and the inner absorber tube, this structure is nonfrangible. The incoming lights are reflected by the parabolic reflector and entered the cavity through its open inlet. Sunlight is then reflected for several times and finally reached the tube receiver at the ellipse focus. There are some studies on optical performance of the solar cavity receivers [10-13]. Very limited studies have been presented to the parameter analysis and optimization of the elliptical cavity tube receiver. Considering this, the PTC with the cavity tube receiver is simulated through using the Monte Carlo method. The
TABLE 1: Parameters of the PTC, cavity, and tube absorber.

\begin{tabular}{lc}
\hline Parameter/unit & Value \\
\hline Major axis/mm & 100 \\
Minor axis/mm & 60 \\
PTC focal distance/mm & 800 \\
Elliptic focal distance/mm & 80 \\
PTC open width/mm & 850 \\
Absorber tube radius/mm & 18 \\
Elliptic cavity open length/mm & 12 \\
\hline
\end{tabular}

parameters of the PTC with the cavity tube receiver are then discussed. A new geometry is finally proposed according to the parameters' analysis results.

\section{Mathematical Models}

To track the solar ray in the PTC, the cross section of a PTC with an elliptical cavity tube receiver is established under the rectangular coordinate system as shown in Figure 2. The major axis, minor axis, and the focal distance of the elliptical cavity are $\mathrm{A}, \mathrm{B}$, and $\mathrm{C}$ in Figure 2, respectively. The open length of the elliptical cavity is $L_{\mathrm{el}}$. The focal distance and open length of the parabolic reflector are $f$ and $L_{\mathrm{pr}}$, respectively. The radius of the tube receiver is $r_{\text {tube }}$. The left focal point of the elliptical cavity is located at the focal point of the PTC. In ideal condition, the sunlight is reflected by the parabolic reflector and concentrated at point $C$. When the sun tracking error occurs, there is a tracking error angle $\alpha$ in the PTC, which causes part of the sunlight not to be reflected into the cavity, that is, Light $S_{1}$ 'BK. There are three representative groups of light paths which can be reflected into the elliptical cavity in Figure 2, namely, the marginal light of the parabolic reflector (i.e., Light $S_{1} B C, S_{2} E C$, and $\mathrm{S}_{3} \mathrm{AC}$ ), the marginal light of the tube receiver (i.e., Light 


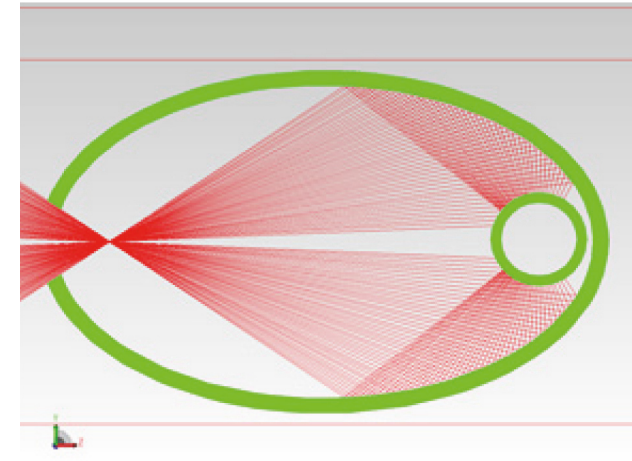

(a)

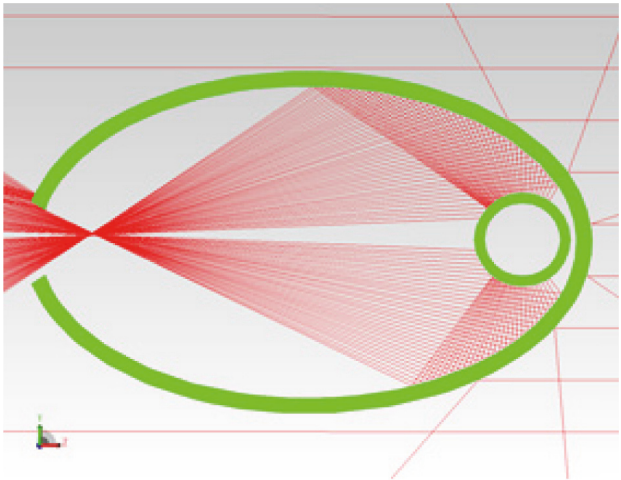

(c)

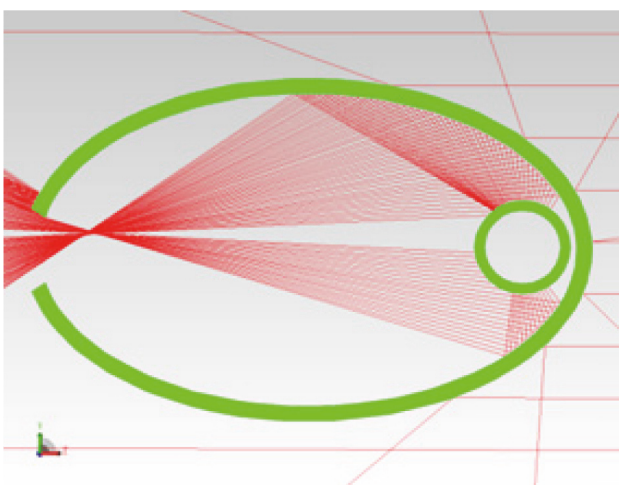

(e)

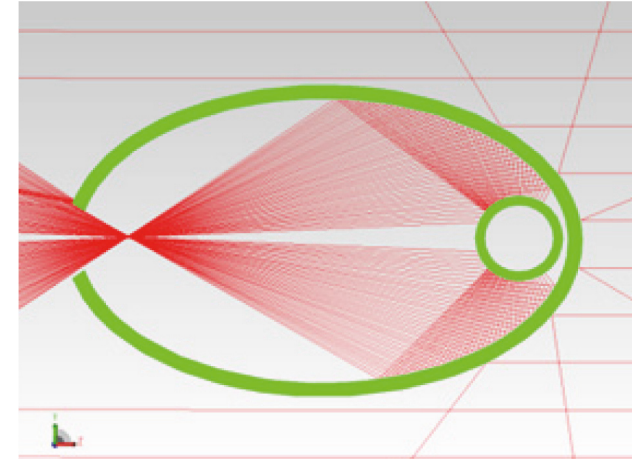

(b)

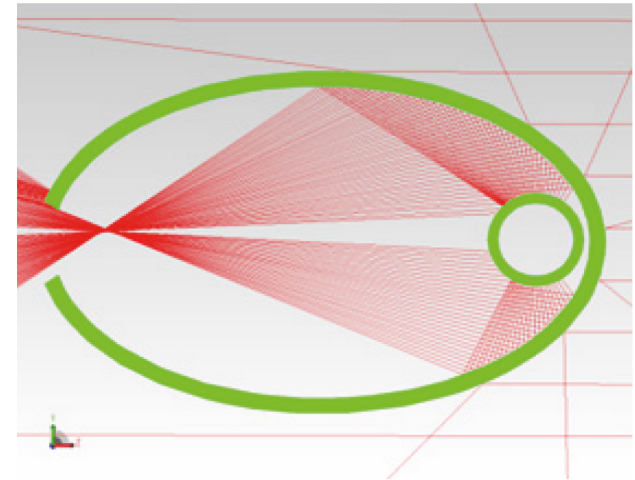

(d)

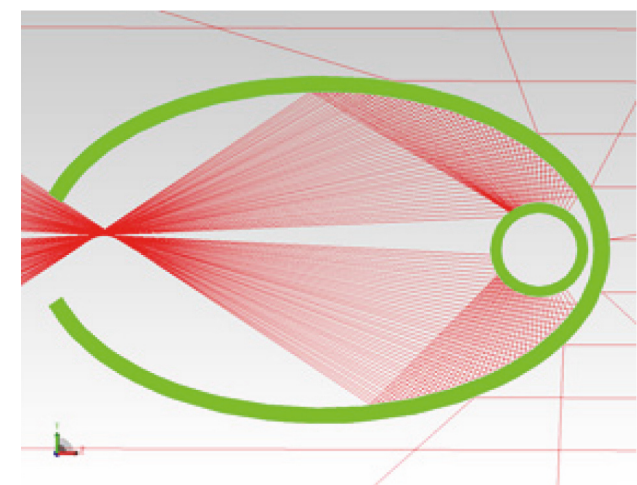

(f)

Figure 3: Light distribution in the elliptical cavity when (a) under ideal condition; (b) $L_{\mathrm{el}}=12 \mathrm{~mm}$ and $\alpha=0.05^{\circ}$; (c) $L_{\mathrm{el}}=12 \mathrm{~mm}$ and $\alpha=0.1^{\circ}$; (d) $L_{\mathrm{el}}=12 \mathrm{~mm}$ and $\alpha=0.15^{\circ}$; (e) $L_{\mathrm{el}}=12 \mathrm{~mm}$ and $\alpha=0.2^{\circ}$; and (f) $L_{\mathrm{el}}=19 \mathrm{~mm}$ and $\alpha=0.2^{\circ}$.

$\mathrm{S}_{3}$ " $\mathrm{AHJ}$ ), and the light with a tracking error angle of $\alpha$ (i.e., Light $\mathrm{S}_{2}{ }^{\prime} \mathrm{EFI}$ ).

2.1. Tube Receiver. According to Figure 2, the elliptic cavity and the parabolic reflector can be expressed as

$$
\begin{aligned}
\frac{(x-f-c)^{2}}{a^{2}}+\frac{y^{2}}{b^{2}} & =1, \\
y^{2} & =4 f x .
\end{aligned}
$$

The marginal light AC can be expressed as

$$
y=k_{1}(x-f)
$$

where

$$
k_{1}=\frac{-B / 2}{\left(B^{2} / 16\right)-f} \text {. }
$$

The angle $\beta$ between the Light $S_{3} A$ and Light $A C$ is

$$
\beta=\arctan k_{1}
$$

The marginal light of the tube receiver $\mathrm{AH}$ can be expressed as 


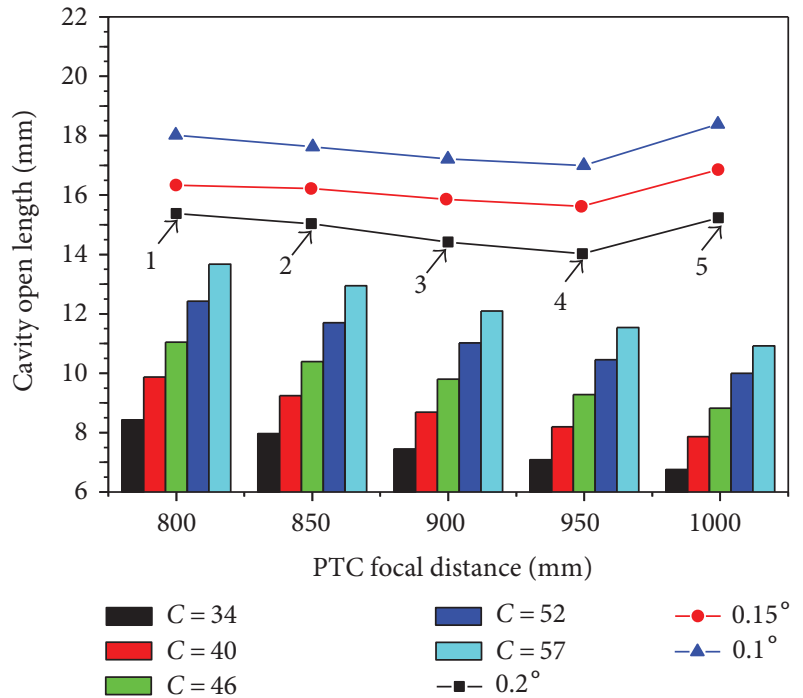

FIGURE 4: Relationship between the PTC focal distance and cavity open length under different concentration ratios and tracking error angles.

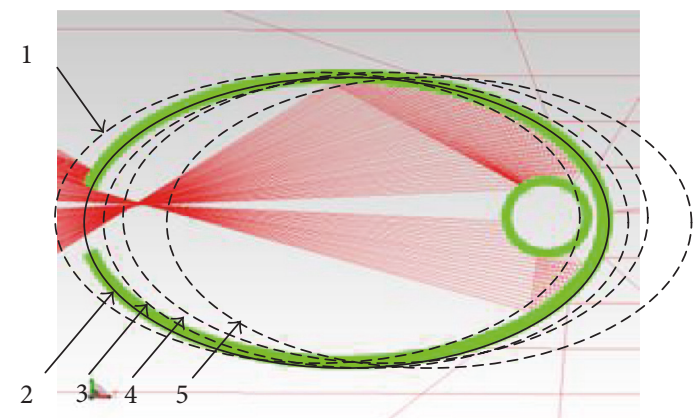

FIGURE 5: Elliptical cavity under different PTC focal lengths when $\alpha=0.2^{\circ}$.

$$
y=k_{2}\left(x-\frac{L_{\mathrm{pr}}^{2}}{16 f}\right)-\frac{L_{\mathrm{pr}}}{2},
$$

where

$$
k_{2}=\tan (\delta+\beta)
$$

Define point $\mathrm{H}$ as $(m, n)$ and the values of $m$ and $n$ can be obtained through (1) and (6). The slope of the tangent light for the tube receiver $\mathrm{HJ}$, which is reflected by the cavity inner surface, is

$$
k_{4}=\frac{k_{2}-k_{3}+k_{3}\left(1+k_{2} k_{3}\right)}{1+k_{2} k_{3}-\left(k_{2}-k_{3}\right) k_{3}},
$$

where $k_{3}$ is the slope of the tangent light:

$$
k_{3}=-\frac{b^{2}(m-f-c)}{a^{2} n} .
$$

Correspondingly, in order to reach the absorber tube inside the cavity, the relationship between the absorber tube radius and the light slopes is

$$
\frac{\left|k_{4}(f+2 c)-k_{4} m+n\right|}{\sqrt{k_{4}^{2}+1}} \leq r_{\text {tube }} .
$$

2.2. Tracking Error Angle. The Light EC can be expressed as

$$
y=k_{5}(x-f) \text {. }
$$

According to (1) and the open length of the elliptical cavity, the Light EFI with a tracking error angle of $\alpha$ is

$$
y=k_{6}\left(x-\sqrt{a^{2}\left(1-\frac{L_{\mathrm{el}}^{2}}{4 b^{2}}\right)}-f-c\right)+\frac{1}{2} L_{\mathrm{el}},
$$

where $k_{5}$ and $k_{6}$ are the slopes of Light EFI and Light EC, respectively.

Correspondingly, in order to enter the elliptical cavity, the relationship between the tracking error angle and the light slopes is

$$
|\alpha| \leq \arctan \frac{k_{5}-k_{6}}{1+k_{5} k_{6}} .
$$

The concentration ratio in the PTC with the elliptical cavity tube receiver is defined as

$$
C=\frac{L_{\mathrm{pr}}-2 b}{2 \pi r}
$$

The Monte Carlo method is utilized to simulate the light distribution in the elliptical cavity. Equations (1)-(13) are then converted into the Fortran codes to determine the configuration sizes of the elliptical cavity and the tube receiver.

\section{Results and Discussion}

3.1. Light Distribution in the Elliptical Cavity. Dimensions of the cavity tube receiver are summarized in Table 1. Light distribution in the elliptical cavity is shown in Figure 3. Figure 3(a) shows the light distribution in the cavity under ideal condition. It is found that the light distribution is longitudinally symmetrical. Taking the above half section as an example, some of the incident sunlight is sheltered by the cavity itself, which causes no light reaching the tube receiver at the left of the tube. The other light is reflected by the parabolic reflector, enters the cavity, and reaches the absorber tube, leading to the increase in the heat flux on the tube. After that, the direct light from the cavity open inlet and the reflected light from the elliptic inner surface are merged, generating a peak on the tube. The other reflected sunlight from the elliptic inner surface then reaches the rest tube surface. No light can reach the range at the right of the tube receiver due to the shelter of the tube itself.

Tracking error usually occurs in practical control of PTCs. Four tracking error angles are then considered in Figures 3(b), 3(c), 3(d), and 3(e), respectively. Due to the 


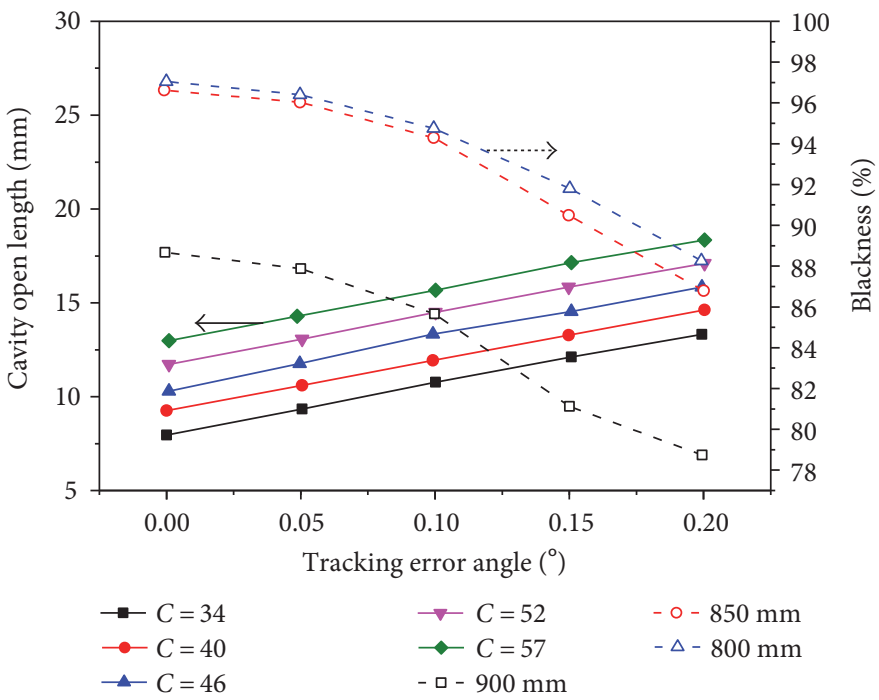

FIGURE 6: The cavity blackness under different tracking error angles, cavity open lengths, concentration ratios, and PTC focal distances.

tracking error, the light distribution on the tube is asymmetrical. Some lights are sheltered by the cavity outer surface. However, the light distribution tendency under ideal condition can also be found in Figures 3(b), 3(c), 3(d), and 3(e), namely, the merged lights directly from the cavity open inlet and reflected from the elliptic inner surface also generate peaks on the above and below half sections of the tubes. On increasing the tracking error angle, more lights are sheltered by the cavity outer surface and less lights reach the below half section of the tube receivers.

The elliptical cavity open length is increased in Figure 3(f). Comparing Figures 3(e) and 3(f), it is found that increasing the cavity open length can allow more lights to enter the cavity. As more sunlight enters, the light distribution on the tube receiver is more close to the ideal condition as shown in Figure 3(a).

3.2. Parameter Analysis. PTC focal distance and cavity open length are two core parameters of the PTC and the cavity tube receiver. Their relationship is discussed in Figure 4. It is found that the PTC focal distance and the tracking error angle have negative correlations with the cavity open length. But the PTC concentration ratio has positive correlation with the cavity open length. When tracking error occurs, on increasing the PTC distance, the cavity open length first decreases and then increases to maintain the concentration ratio. This can be explained by Figure 5 . Taking the condition of $\alpha=0.2^{\circ}$ as an example, five elliptical cavity locations are shown in Figure 5. It is found that on increasing the PTC focal distance, the elliptical cavity is moved toward right. As the reflected light is first concentrated toward the focal point and then diverges in the cavity, the cavity open length needs to first decrease then increase to allow the light to enter the cavity.

For the cavity tube receiver, the blackness, which is the percentage of the sunlight on the tube receiver surface to the total incident sunlight, is proposed to evaluate the cavity performance. The cavity open length and cavity blackness under different tracking error angles are shown in Figure 6. It is found that, to a specific PTC and cavity tube receiver, increasing the tracking error angles would decrease the cavity blackness; and in order to maintain the concentration ratio, the cavity open length needs to be enlarged. Under a specific tracking error angle, increasing the PTC focal distance would decrease the cavity blackness. The reason is that more sunlight is sheltered by the cavity outer surface as indicated in Figures 3(b), 3(c), 3(d), and 3(e). Also, when the PTC concentration ratio increases, for example the PTC width increases, the cavity open length needs to be increased to allow more light to transfer into the cavity as indicated by angle $\beta$ in Figure 2 .

3.3. Elliptical Cavity Optimization. According to the discussion above, a flat plate reflector is added at the cavity inlet to enhance the cavity tube receiver performance. The geometry of the proposed cavity is shown in Figure $7(\mathrm{a})$, and the top flat plate reflector can be described as

$$
y=-\tan \beta(x+c) .
$$

The light distribution in the cavity is shown in Figure 7(b). It is found that the sheltered sunlight is reflected by the flat plate, which can finally reach the tube receiver. The cavity darkness of the new cavity receiver under different tracking error angles and PTC focal distances is shown in Figure 8. According to Figures 3, 6, 7(b), and 8, it is found that introducing the flat plate can largely increase the cavity darkness. The cavity darkness decreases when the tracking error angle increases. But introducing the flat plate reflector breaks the monotonic relationships of the cavity darkness under different PTC focal distances. Moving the elliptical cavity along the long axis direction leads to the incident sunlight reflected by the flat plate under different incident angles, which generates different multireflections inside the cavity, and finally leads to the curves in Figure 8. 


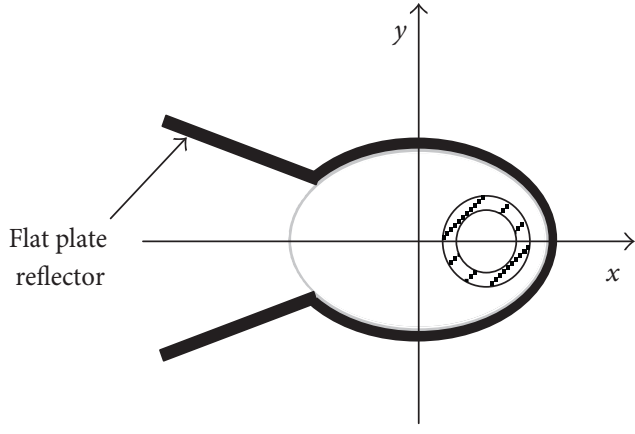

(a)

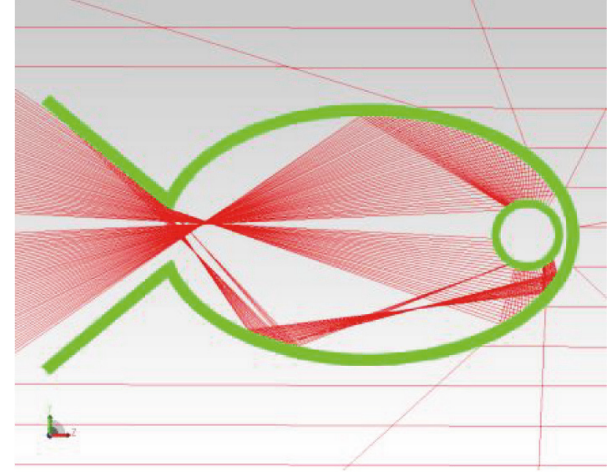

(b)

Figure 7: (a) Newly proposed elliptical cavity geometry and (b) light distribution in the cavity.

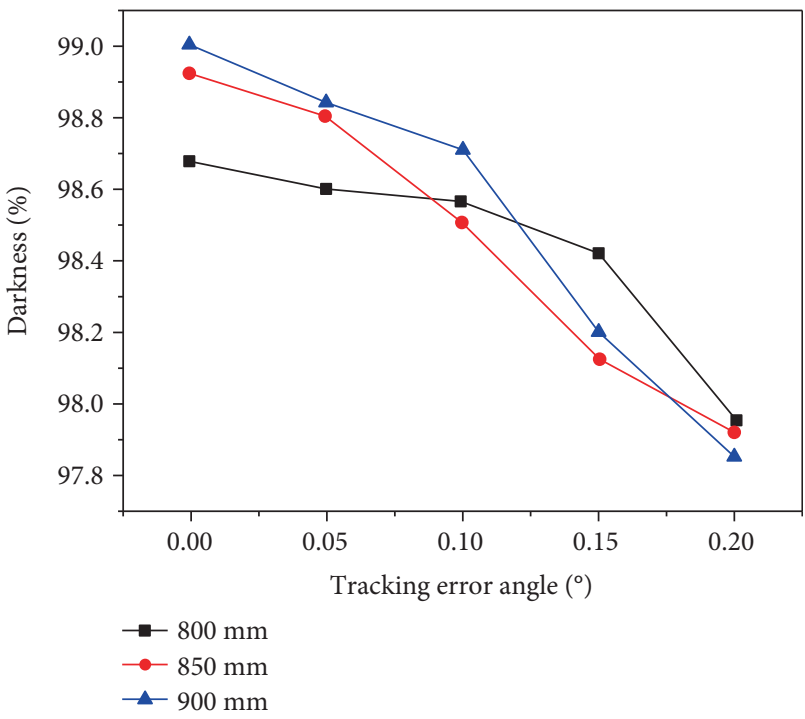

FIGURE 8: Darkness of the newly proposed cavity receiver under different tracking error angles and PTC focal distances.

\section{Conclusions}

Solar parabolic trough collectors (PTC) are the most widely accepted solar concentration style. The PTC with an elliptical cavity tube receiver has not been well discussed in the literature. In the present study, the light distribution in the cavity under different tracking error angles and PTC configurations are analyzed. A new elliptical cavity geometry is proposed and analyzed. The following conclusions are obtained through this study:

(1) The light distribution on the tube receiver is asymmetrical. On increasing the tracking error angle, more lights are sheltered by the cavity outer surface and less lights reach the below section of the tube receivers.

(2) The PTC focal distance and the tracking error angle have negative correlations with the cavity open length, whereas the PTC concentration ratio has positive correlation with the cavity open length. On increasing the PTC focal distance, the cavity open length needs to first decrease and then increase to maintain the concentration ratio.

(3) Increasing the tracking error angles would decrease the cavity blackness. Increasing the PTC focal distance would decrease the cavity blackness. Introducing a flat plate reflector at the elliptical cavity open inlet leads to multireflections inside the cavity, which can largely increase the cavity darkness.

\section{Conflicts of Interest}

The authors declare that they have no conflicts of interest.

\section{Acknowledgments}

This research was funded by the National Natural Science Foundation of China (no.: 51506043), the Fundamental Research Funds for the Central Universities (no.: 2014B19714), and the Applied Basic Research Plan of Changzhou City (no.: CJ20160043).

\section{References}

[1] V. K. Jebasingh and G. M. J. Herbert, "A review of solar parabolic trough collector," Renewable and Sustainable Energy Reviews, vol. 54, pp. 1085-1091, 2016.

[2] J. J. Serrano-Aguilera, L. Valenzuela, and J. FernandezReche, "Inverse Monte Carlo Ray-Tracing method (IMCRT) applied to line-focus reflectors," Solar Energy, vol. 124, pp. 184-197, 2016.

[3] Z. D. Cheng, Y. L. He, J. Xiao, Y. B. Tao, and R. J. Xu, "Three-dimensional numerical study of heat transfer characteristics in the receiver tube of parabolic trough solar collector," International Communications in Heat and Mass Transfer, vol. 37, no. 7, pp. 782-787, 2010.

[4] Z. D. Cheng, Y. L. He, F. Q. Cui, B. C. Du, Z. J. Zheng, and $\mathrm{Y} . \mathrm{Xu}$, "Comparative and sensitive analysis for parabolic trough solar collectors with a detailed Monte Carlo ray-tracing optical model," Applied Energy, vol. 115, no. 4, pp. 559-572, 2014.

[5] G. M. Giannuzzi, C. E. Majorana, A. Miliozzi, V. A. Salomoni, and D. Nicolini, "Structural design criteria for steel components 
of parabolic-trough solar concentrators," Journal of Solar Energy Engineering, vol. 129, no. 4, pp. 382-390, 2007.

[6] H. Liang, S. You, and H. Zhang, "Comparison of three optical models and analysis of geometric parameters for parabolic trough solar collectors," Energy, vol. 96, pp. 37-47, 2016.

[7] Z. Cheng, Y. He, and D. Baochun, "Geometric optimization on optical performance of parabolic trough solar collector systems using particle swarm optimization algorithm," Applied Energy, vol. 148, pp. 282-293, 2015.

[8] P. M. Zadeh, T. Sokhansefat, A. B. Kasaeian, F. Kowsary, and A. Akbarzadeh, "Hybrid optimization algorithm for thermal analysis in a solar parabolic trough collector based on nanofluid," Energy, vol. 82, pp. 857-864, 2015.

[9] G. Kumaresan, R. Sridhar, and R. Velraj, "Performance studies of a solar parabolic trough collector with a thermal energy storage system," Energy, vol. 47, no. 1, pp. 395-402, 2012.

[10] V. S. Reddy, S. C. Kaushik, and S. K. Tyagi, "Exergetic analysis and performance evaluation of parabolic trough concentrating solar thermal power plant (PTCSTPP)," Energy, vol. 39, no. 1, pp. 258-273, 2012.

[11] F. Chen, M. Liu, P. Zhang, and X. Luo, "Thermal performance of a novel linear cavity absorber for parabolic trough solar concentrator," Energy Conversion and Management, vol. 90, pp. 292-299, 2015.

[12] F. Chen, M. Liu, R. H. E. Hassanien et al., "Study on the optical properties of triangular cavity absorber for parabolic trough solar concentrator," International Journal of Photoenergy, vol. 2015, Article ID 895946, 9 pages, 2015.

[13] W. Gao, G. Q. Xu, T. T. Li, and H. W. Li, "Modeling and performance evaluation of parabolic trough solar cavity-type receivers," International Journal of Green Energy, vol. 12, no. 12, pp. 1263-1271, 2015. 

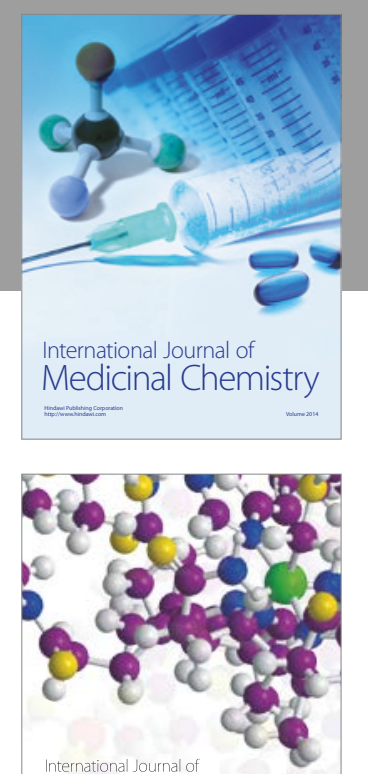

Carbohydrate Chemistry

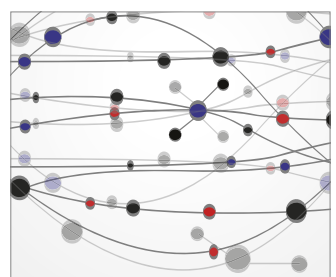

The Scientific World Journal
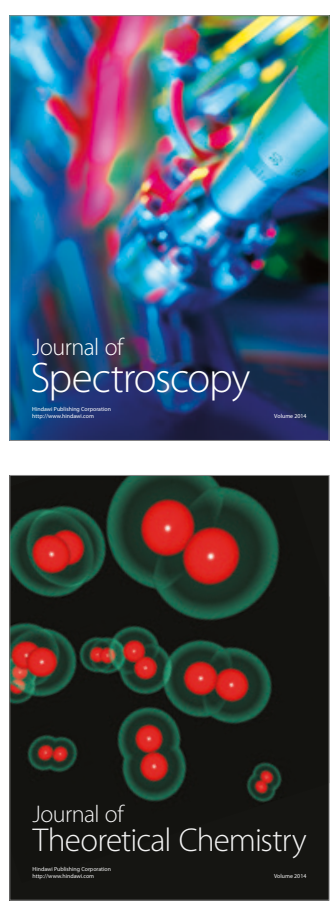
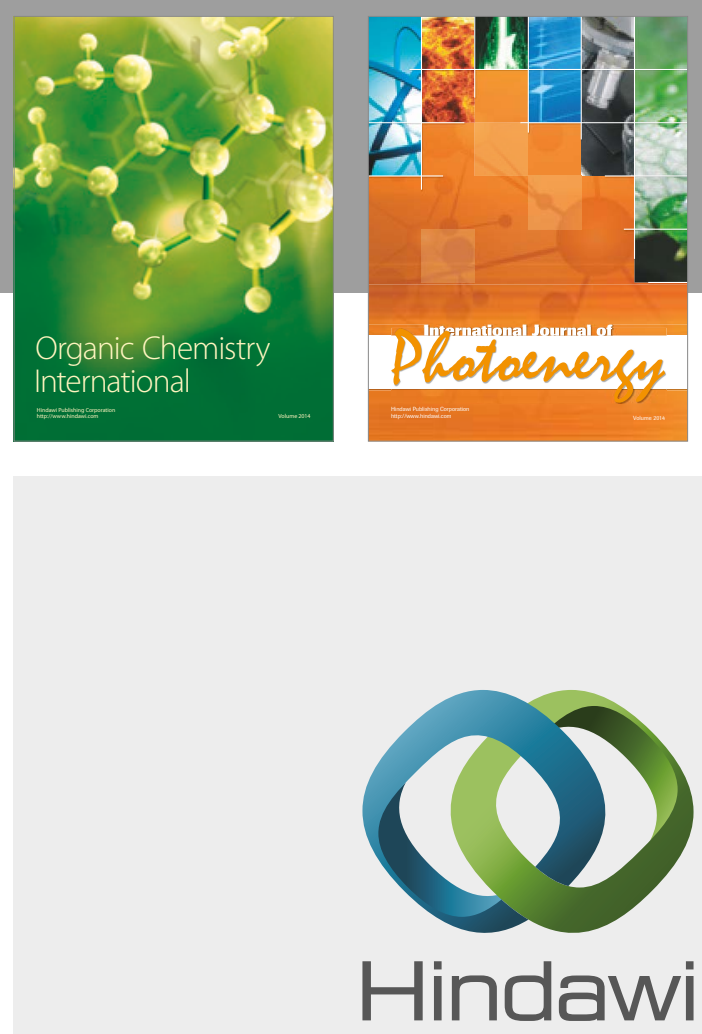

Submit your manuscripts at

https://www.hindawi.com

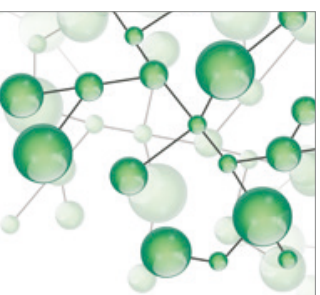

International Journal of

Inorganic Chemistry

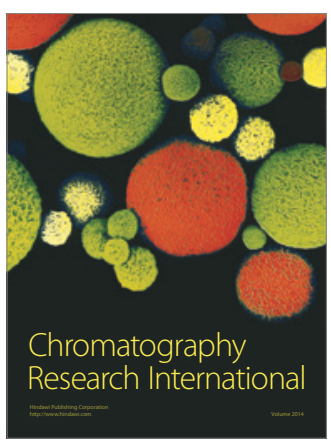

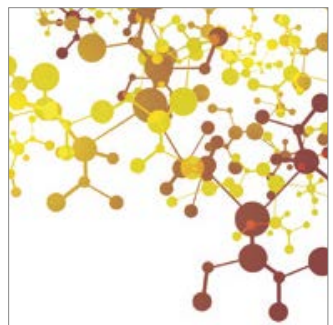

Applied Chemistry
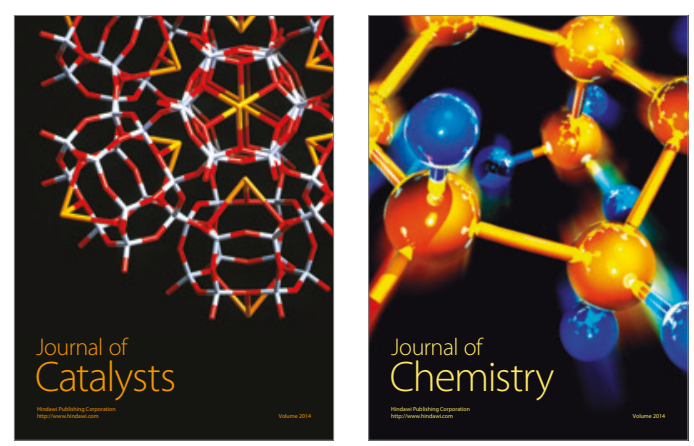
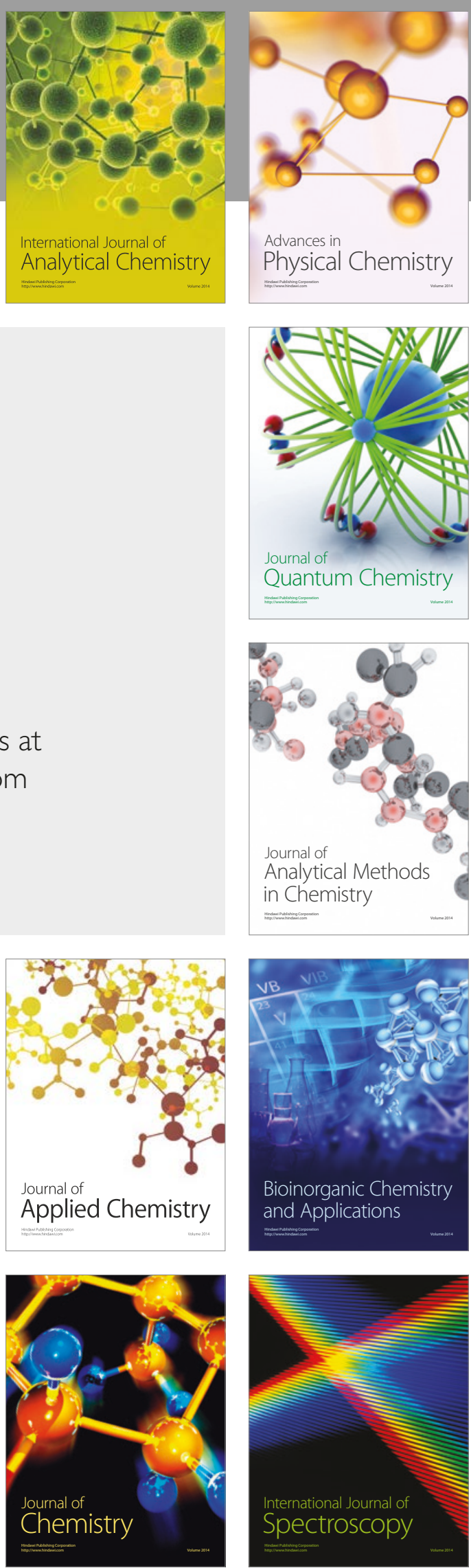